\title{
Weightbearing ovine osteochondral defects heal with inadequate subchondral bone plate restoration: implications regarding osteochondral autograft harvesting
}

\author{
Tomasz L. Nosewicz $\cdot$ Mikel L. Reilingh • \\ C. Niek van Dijk • Georg N. Duda • \\ Hanna Schell
}

Received: 7 July 2011/Accepted: 9 December 2011/Published online: 21 December 2011

(C) The Author(s) 2011. This article is published with open access at Springerlink.com

\begin{abstract}
Purpose It is unknown what causes donor site morbidity following the osteochondral autograft transfer procedure or how donor sites heal. Contact pressure and edge loading at donor sites may play a role in the healing process. It was hypothesized that an artificially created osteochondral defect in a weightbearing area of an ovine femoral condyle will cause osseous bridging of the defect from the upper edges, resulting in incomplete and irregular repair of the subchondral bone plate.

Methods To simulate edge loading, large osteochondral defects were created in the most unfavourable weightbearing area of 24 ovine femoral condyles. After killing at 3 and 6 months, osteochondral defects were histologically and histomorphometrically evaluated with specific attention to subchondral bone healing and subchondral bone plate restoration.

Results Osteochondral defect healing showed progressive osseous defect bridging by sclerotic circumferential bone apposition. Unfilled area decreased significantly from 3 to 6 months $(P=0.004)$, whereas bone content increased (n.s.). Complete but irregular subchondral bone plate restoration occurred in ten animals. In fourteen animals, an
\end{abstract}

Study performed at the Julius Wolff Institute and Center for Musculoskeletal Surgery, Charité Universitätsmedizin Berlin, Germany.

T. L. Nosewicz $(\bowtie) \cdot$ G. N. Duda $\cdot$ H. Schell

Julius Wolff Institute and Center for Musculoskeletal Surgery, Charité Universitätsmedizin Berlin, Augustenburger Platz 1,

Forum 4, Postbox 24, 13353 Berlin, Germany

e-mail: t.nosewicz@gmail.com

T. L. Nosewicz · M. L. Reilingh · C. N. van Dijk Department of Orthopaedic Surgery, Academic Medical Center, Meibergdreef 9, 1105 AZ Amsterdam, The Netherlands incomplete subchondral bone plate was found. Further common findings included cavitary lesion formation, degenerative cartilage changes and cartilage and subchondral bone collapse.

Conclusions Osteochondral defect healing starts with subchondral bone plate restoration. However, after 6 months, incomplete or irregular subchondral bone plate restoration and subsequent failure of osteochondral defect closure is common. Graft harvesting in the osteochondral autograft transfer procedure must be viewed critically, as similar changes are also present in humans.

Level of evidence Prognostic study, Level III.

Keywords Donor site morbidity - Osteochondral autograft transfer - Subchondral bone plate

\section{Introduction}

The osteochondral autograft transfer (OATS) procedure is commonly used for resurfacing articular cartilage defects [22, 25, 38, 39]. In OATS, the lateral and medial trochlea $[15,16]$, femoral condyles $[12,13]$, as well as the intercondylar notch [16] are common harvesting sites. Graft size varies between 2.7 and $10 \mathrm{~mm}$ in diameter and up to $30 \mathrm{~mm}$ in depth $[12,13,15,16]$. Through graft harvesting, a full thickness osteochondral defect (OCD) is artificially created in a healthy joint surface. Although small traumatic OCDs do not cause many problems [25, 34], the defects created through harvesting are considerably larger [12, 15].

Donor site morbidity $[12,13,22,26,29,37]$ is a frequent complication following the OATS procedure. Patients may present with pain, decreased knee function and knee instability [12, 22, 26, 29, 37]. To minimize donor site morbidity, investigators state to harvest 
osteochondral grafts from less or even non-weightbearing areas of the femoral condyle [13, 17, 22]. However, biomechanical studies have shown significant contact pressures in all recommended donor sites of the human knee $[1,8,10,35]$. Surprisingly, little research has been performed in the clinical outcome of these donor sites. Following graft harvesting, Valderrabano et al. [37] found that donor site morbidity was associated with a discontinued subchondral bone plate on MRI.

It is furthermore unknown how donor sites heal. An animal study showed subchondral bone plate restoration by circumferential osseous defect closure as a potential mechanism [14]. The stimulus for such osseous bridging could be increased edge loading at the weightbearing areas [19], coupled to a physiological healing response guided by Wolff's law [4].

In the present study, the healing of full thickness weightbearing ovine OCDs was investigated by comparing these to the native situation. It was hypothesized that an artificially created OCD in a weightbearing area of the femoral condyle will cause osseous bridging of the defect from the upper edges, resulting in incomplete and irregular repair of the subchondral bone plate. In order to simulate the effect of significant edge loading [1, 8, 10, 19, 35] on osteochondral healing, the defects were specifically created in the most unfavourable location in the weightbearing portion of the femoral condyle.

\section{Materials and methods}

In this study, 24 skeletally mature female Merino-mix sheep were used. All animal experiments were carried out according to the policies and principles established by the Animal Welfare Act, the NIH Guide for Care and Use of Laboratory Animals and the National Animal Welfare Guidelines. The experiments were approved by the local legal representative. All operations were performed under general anaesthesia and by the same surgeon. The sheep were initially sedated with $0.5 \mathrm{~g} / 50 \mathrm{~kg}$ bodyweight intravenously administered thiopental-sodium (Trapanal $2.5 \mathrm{~g}$, Altana Pharma Deutschland GmbH, Konstanz, Germany). Anaesthesia was maintained by inhalation of $1.5 \%$ isoflurane in $33 \%$ oxygen and $66 \%$ nitrogen. Analgesia was provided by administering a $0.025 \mathrm{mg}$ fentanyl bolus (Fentanyl-Janssen, $0.5 \mathrm{mg}$, Janssen-Cilag GmbH, Neuss, Germany) every $45 \mathrm{~min}$ for the entire duration of the operative procedure. A single shot prophylaxis of $2.2 \mathrm{~g}$ of amoxicillin and clavulanate (Augmentin $2.2 \mathrm{~g}$, GlaxoSmithKline GmbH \& Co Kg, Munich, Germany) was given intra operatively. All sheep were operated on the left hind limb. The knee joint was opened by an anteromedial arthrotomy [2, 31]. In the weightbearing areas of the femoral condyles, OCDs were created using cylindrical hollow punches $\left(\right.$ SDS $^{\circledR}$-System, Zimmer Germany GmbH, Freiburg, Germany). The defects were evenly created in the center of the lateral or the medial femoral condyle. This was done to exclude any potential influence of defect location.

The defects measured $10 \mathrm{~mm}$ in height and $8.3 \mathrm{~mm}$ in diameter. The osteochondral autografts were thereafter press-fitted in recipient sites on the contralateral condyle of the same knee by using the OATS procedure. The results of this OATS procedure have been previously reported by Kleemann et al. [18].

The right hind limb served as intact native control. Postoperative healing times were 3 and 6 months $(n=12$ per time point). The killing of animals at 3 and 6 months was specifically chosen as Jackson et al. at these time points showed crucial changes in OCD healing, consisting of bone resorption, articular cartilage and subchondral bone collapse and cavitary lesion formation [14].

Postoperatively, the animals were allowed immediate free movement. The general condition and the surgery wounds were checked every day until the sutures were removed at day 10 .

After killing and exarticulation of the operated and native hind limbs, the OCD and native areas were sawed in sagittal plane. Care was taken to obtain a native sample from a position, which resembled most the contralateral operated area. One half of each sample was dehydrated and embedded in methylmethacrylate (Technovit 9100, Kulzer, Germany) and cut (Leica Microsystems Nussloch GmbH, Nussloch, Germany) into 6- $\mu$ m-thick sections. The other half was decalcified and embedded in paraffin and cut (Leica Microsystems Nussloch GmbH, Nussloch, Germany) into $4-\mu m$-thick sections. The methylmethacrylate was dissolved with methoxymethyl acetate, followed by incubation in alcohol for rehydration. The samples were stained with Safranin Orange/Van Kossa and Safranin Orange/Fast Green. Paraffin sections were deparaffinized in xylene, rehydrated in alcohol and afterwards stained with tartrate-resistant acid phosphatase (TRAP) in order to monitor bone remodelling activity.

Semi-quantitative histological evaluation was performed on Safranin Orange/Van Kossa and Safranin Orange/Fast Green samples under light microscopy (magnification 10, 20 and 50 fold). Cartilage and subchondral bone plate repair (score A) was assessed by using a modification of the score by Frenkel et al. [7] (Table 1), whereas subchondral bone repair (score B) was assessed with a modification of the score by Mainil-Varlet [21] (Table 2). Native samples received the full amount of points, respectively, 27 and 9 for score $\mathrm{A}$ and $\mathrm{B}$. The quantification of osteoclasts was performed under light microscopy (magnification 10-fold) in a region of interest (ROI), 
Table 1 Histological grading score A (cartilage and subchondral bone plate repair)

Category (points)

Percentage fibrocartilaginous articular cartilage

$80-100 \%(8)$

60-80\% (6)

$40-60 \%$ (4)

$20-40 \%$ (2)

$0-20 \%(0)$

Structural characteristics

Surface regularity

Smooth and intact (2)

Fissures (1)

Fibrillation, severe disruption (0)

Structural integrity

Normal (2)

Slight disruption, including cysts (1)

Severe lack of integration (0)

Cartilage thickness ( $\%$ of surrounding normal cartilage)

$100 \%$ (2)

$50-100 \%$ or more (1)

0-50\% (0)

Bonding to adjacent cartilage

Bonded to both sides (2)

Bonded to one side; partially bonded to both sides (1)

Not bonded (0)

Cellular changes of degeneration

Normal cellularity, no chondrocyte clustering (2)

Light hypocellularity, $<25 \%$ chondrocyte clustering (1)

Moderate hypocellularity, $>25 \%$ chondrocyte clustering (0)

Cellular changes of degeneration in adjacent cartilage

Normal cellularity, no chondrocyte clustering, normal staining (3)

Normal cellularity, few chondrocyte clustering, moderate staining (2)

Mild or moderate hypocellularity, slight staining (1)

Severe hypocellularity, many chondrocyte clustering, no staining (0)

Reconstruction of the subchondral bone plate

Complete reconstruction (2)

More than 50\% reconstruction (1)

$50 \%$ or less reconstruction $(0)$

Connection between cartilage and subchondral bone

Fully and not interrupted (2)

$<100 \%$ but $>50 \%$ (1)

$<50 \%$ (0)

Matrix staining (Safranin $O$ )

$>80 \%$ homogeneous positive staining (2)

$40-80 \%$ homogeneous positive staining (1)

$<40 \%$ homogeneous positive staining (0)

Total maximum points score $A=27$
Table 2 Histological grading score B (subchondral bone repair)

Category (points)

Filling of defect area

$91-110 \%$ (4)

$76-90 \%(3)$

$51-75 \%(2)$

26-50\% (1)

$<25 \%(0)$

Lateral osseous integration

Bonded at both sides (2)

Bonded at one side, partially at both sides (1)

No bonding (0)

Basal integration

91-100\% (3)

$70-90 \%$ (2)

50-70\% (1)

$<50 \%$ (0)

Total maximum points $B=9$

matching in size the original OCD [31]. TRAP-positive cells with at least two nuclei which were situated on a bone surface were counted as osteoclasts. The number of osteoclasts was thereafter referenced to $50 \mathrm{~mm}^{2}$ of mineralized bone. The amount of tissue was obtained from the image analysis program.

Samples stained with Safranin O/Van Kossa and Safranin O/Fast Green were digitally photographed and histomorphometrically analysed using a Zeiss KS 400 image analysis workstation (Carl Zeiss Vision, Oberkochen, Germany). A computerized ROI, matching in size the initial OCD, was used in both groups and placed over the digital photographs in order to standardize the analysis procedure. Histomorphometric analysis consisted of quantifying the amounts of mineralized bone and unfilled area in percent of the total ROI area. The unfilled area was defined as the area between the extrapolated curvature of the recipient hyaline cartilage and the first tissue encountered below this curvature.

Statistical analysis

Statistical comparison of the operation procedure between both groups and between time points was performed with the Mann-Whitney $\mathrm{U}$ test. To compare grafted and native samples within the same operation group and time variable, the Wilcoxon test for 2 related samples was performed. The level of significance was set at $P<0.05$. Statistical analysis was performed with SPSS 17.0 (SPSS Inc. Chicago, USA). 


\section{Results}

Descriptive histological analysis

Healing in all samples after 3 and 6 months showed progressive circumferential bone apposition aimed at subchondral bone plate restoration from the upper edges, coupled to cavitary lesion formation and cartilage and subchondral bone collapse. Degenerative changes, i.e., chondrocyte cluster formation and moderate staining reflecting proteoglycan loss, were present in both reparative and adjacent native cartilage.

Healing was characterized by chronological progression in 4 stages (Fig. 1). After 3 months, stages 1, 2, 3 and 4 were represented 3, 3, 4 and 2 times, respectively; after 6 months, 0, 2, 2 and 8 times. Complete but irregular subchondral bone plate restoration was found in ten samples (Fig. 2). In these stage 4 samples, the central area showed an empty cavity ( $n=3$ ), a cavity lined by fibrous tissue and a centrally placed cystic vacuolization $(n=3)$, a cavity fully filled with fibrous tissue $(n=2)$ or a central area almost totally filled with dense bone $(n=2)$. These latter two samples without cavitary lesion formation centrally showed fibrocartilage remnants and thus were not fully filled with mineralized bone.

\section{Histology and histomorphometry}

While score A improved significantly from 3 to 6 months $(P=0.004)$, score B did not. OCDs at both time points showed significantly more osteoclasts than native samples $(P<0.05)$. Whereas the evident increase (n.s.) in mineralized bone in the OCDs at 6 months resulted in a situation similar to native, the significant decrease $(P=0.004)$ in unfilled area did not $(P=0.002)$ (Table 3$)$.

\section{Discussion}

The most important findings in the present study are that OCDs heal with irregular and incomplete restoration of the subchondral bone plate, native and reparative cartilage degeneration, collapse of sclerotic subchondral bone and cartilage, increase in the primary defect through osseous erosion and the formation of a central cavitary lesion. Similar changes were seen by Jackson et al. [14], Gotterbarm et al. [9] and Schlichting et al. [32].

Whereas at 3 months, $17 \%$ of the samples (2/12) showed osseous defect bridging, at 6 months, this was $67 \%$ $(8 / 12)$. Furthermore, the decrease in unfilled area suggests that the defects after 6 months were approaching the level of the adjacent native cartilage. Although this implies progressive subchondral bone plate restoration, Jackson et al. [14] after 1 year and Valderrabano et al. [37] after 72 months showed that incomplete subchondral bone plate restoration is common.

Our findings show that healing following OCD creation in weightbearing areas results in circumferential subchondral bone plate restoration from the upper defect edges $[14,20]$. This was confirmed by the absence of bone formation from the depth of the defect as previously described by Jackson et al. [14], resulting in a cavitary lesion. It may be speculated that subchondral bone plate restoration results from a physiological, Wolff's law driven process under the influence of increased edge loading [4, 19].

Defect bridging, as previously shown by Duda et al. [6] and in the present study, reflected by the improvement in score $\mathrm{A}$ and the significant decrease in unfilled area from 3 to 6 months, most probably is carried out to restore the normal biomechanical properties of the joint, preventing adjacent cartilage overload [3, 19, 38]. Bone apposition is aided by the formation of circumferential fibrous strands, serving as a scaffold for pluripotent mesenchymal stem cells [33] and subsequent osteoblasts [6]. Initially, there is collapse of this newly formed circumferential subchondral bone and articular cartilage [3, 9, 14], most probably due to significant contact pressure [1, 8, 10, 35] and failed peripheral bone support [14]. Progressive bone apposition eventually results in an aperture through which synovial fluid can enter, resulting in fluid pressurization [20, 25, 38]. Due to a local stress-shielding effect of the pressurized fluid, net bone resorption occurs at the trabeculae surrounding the fluid [5]. This explains the initial osseous erosion and subsequent increase in the initial defect as seen in our investigation and Jackson's study [14]. In our study, we found an increased amount of osteoclasts as a sign of active bone resorption. As such, score B did not improve from 3 to 6 months, despite the paradoxical increase in mineralized bone due to bone sclerosis, which has also been reported by Schlichting et al. [32].

In the study by Valderrabano et al. [37], at a mean follow-up of 72 months, all 12 patients showed cavitary lesions, but only half showed donor site morbidity. Moreover, donor site morbidity was associated with incomplete subchondral bone plate restoration; 4 out of 6 patients with donor site morbidity showed an discontinued subchondral bone plate on MRI, whereas 5 out of 6 patients without donor site morbidity showed an intact subchondral bone plate. This stresses the importance of subchondral bone plate regeneration following grafting [20, 23, 25, 27] and suggests that loading of an incompletely formed but highly innervated subchondral bone plate elicits pain [5, 20, 38].

Finally, cartilage degeneration [37] may also play a role in donor site morbidity. The significant edge loading in large OCDs [19] stimulates excessive bone formation [4] and results in sclerotic subchondral bone plate restoration 

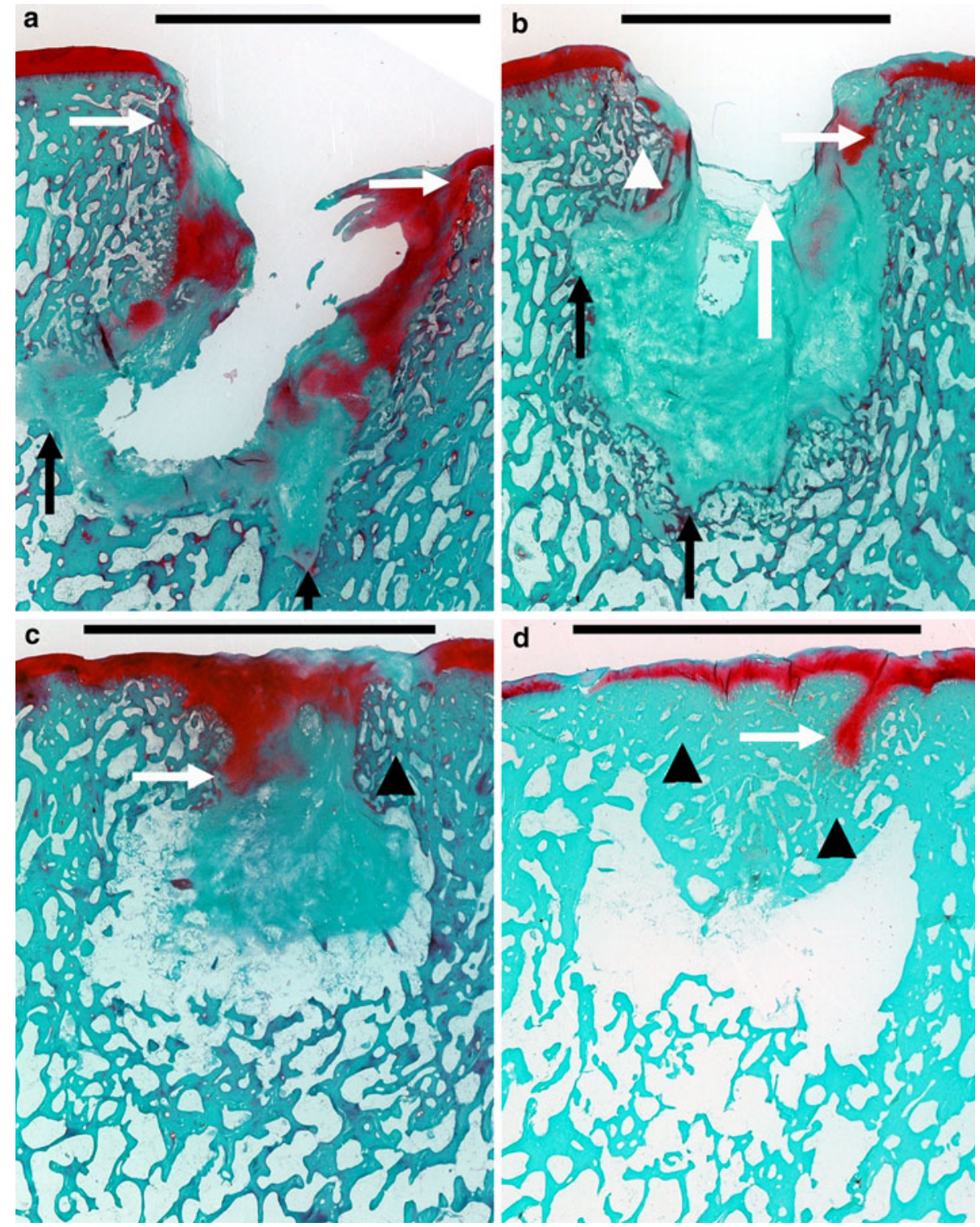

Fig. 1 Subchondral bone plate restoration stages. In these Safranin $\mathrm{O} /$ Fast Green stained samples, mineralized bone and fibrous tissue appear bluish green, whereas (fibro)cartilage appears red. Scale bars represent the diameter of the initial osteochondral defect. a In stage 1 $(n=3)$, the defect was continuous with the synovial cavity, with no signs of defect bridging. Notice the extensive (fibro)cartilage collapse (white horizontal arrows) and erosion of bone outside the perimeters of the initially created defect (black vertical arrows). b In stage 2 $(n=5)$, fibrous tissue bridging or minor circumferential subchondral bone plate restoration was present. The main difference with stage 1 was the formation of fibrous strands in an attempt to partially close the created defect (white vertical arrow). Again, collapse of (fibro)cartilage (white horizontal arrow) and subchondral bone (white arrowhead), coupled to osseous erosion (black vertical arrows), was

$[14,32]$. An increase in bone density leads to mechanical stiffening of the subchondral bone, causing increased mechanical stress in the overlying articular cartilage [28]. This could explain the degenerative chondrocyte clustering seen frequently. Already, the first signs of a central irregularly shaped cavitary lesion were seen. This lesion was either empty or filled with connective tissue. In some samples, central vacuolization of the fibrous tissue was present. c In stage $3(n=6)$, there was still inward collapse of subchondral bone and (fibro)cartilage. Progressive subchondral bone plate restoration was observed. Notice the evident subchondral bone sclerosis (black arrowhead). The central cavitary lesion, which was nearly closed, either was lined with fibrous tissue or showing exposed bone. d In stage $4(n=10)$, there was complete sclerotic osseous bridging of the defect, with mostly a concave fibrocartilaginous articular surface, and a central cavitary lesion which was either empty or filled with fibrous tissue. Again, cartilage flow and subchondral bone sclerosis were present

$[9,14]$ and the formation of a degenerative zone of influence [14] in the adjacent, native cartilage.

As non-weightbearing areas in the knee do not exist [1, $8,10,35]$, research in how to enhance OCD healing is 
Fig. 2 Subchondral bone plate restoration in stage 4 Safranin $\mathrm{O} / \mathrm{Van}$ Kossa stained samples. Mineralized bone appears black, and (fibro)cartilage and fibrous tissue appear red. Scale bars represent the diameter of the initial osteochondral defect. The subchondral bone plate was characterized by irregular restoration, compromised by fibrocartilage flow (white arrows), the underlying cavitary lesion (black arrow) and subchondral bone plate advancement (arrowheads)

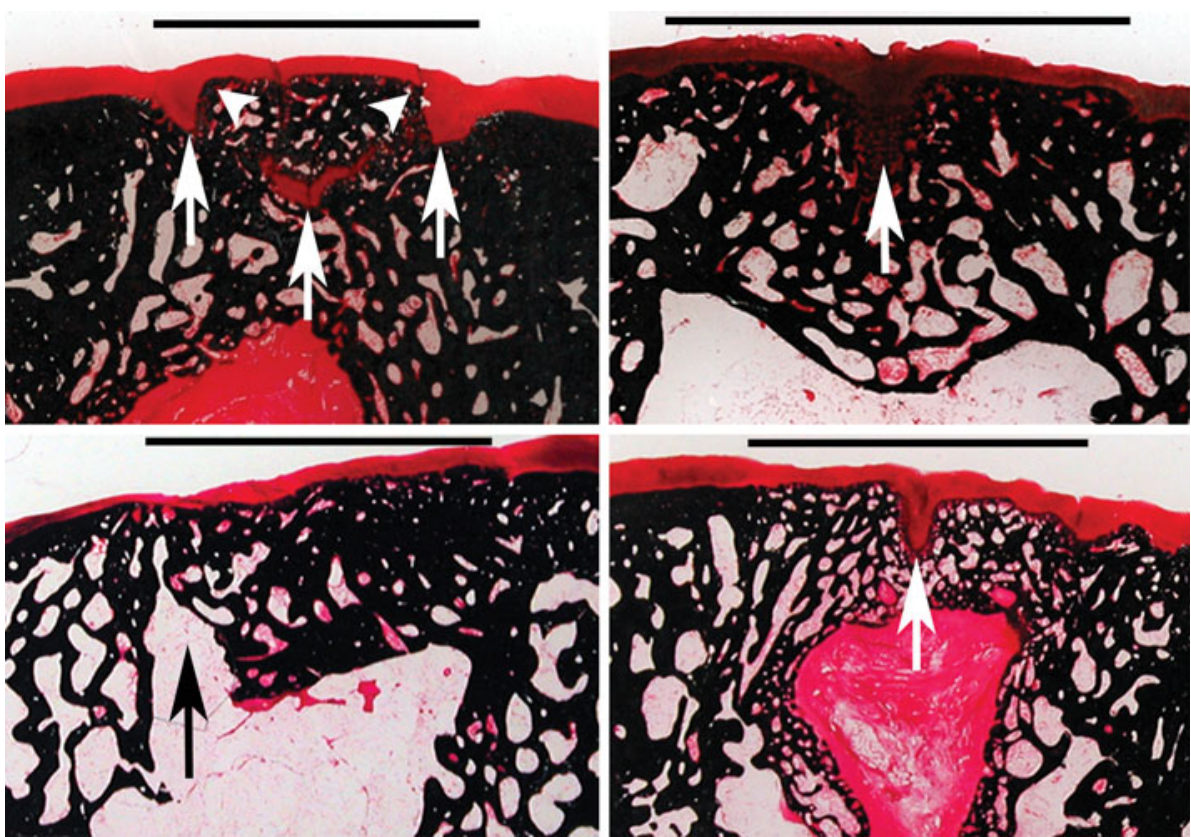

Table 3 Histological and histomorphometric results for the native and osteochondral defect (OCD) group

\begin{tabular}{|c|c|c|c|c|c|c|}
\hline \multirow{2}{*}{$\begin{array}{l}\text { Time } \\
\text { (months) }\end{array}$} & \multirow[t]{2}{*}{ Group } & \multicolumn{3}{|l|}{ Histology } & \multicolumn{2}{|l|}{ Histomorphometry } \\
\hline & & Score A & Score B & OC's & Unfilled area & Mineralized bone \\
\hline \multirow[t]{2}{*}{3} & Native & $27 *$ & $9 *$ & $4 \pm 6(0-18)$ & $0 \S$ & $46.8 \pm 8.0(36.4-58.3)$ \\
\hline & OCD & $3.8 \pm 3.4(1-11) \dagger ; \uparrow$ & $3.8 \pm 3.3(0-9) \ddagger$ & $79 \pm 50(11-156) \ddagger$ & $16.1 \pm 13.5(2.0-41.7) \dagger, \dagger$ & $25.3 \pm 18.4(5.0-54.7) \ddagger$ \\
\hline \multirow[t]{2}{*}{6} & Native & $27 *$ & $9 *$ & $2 \pm 4(0-11)$ & $0 \S$ & $43.9 \pm 6.5(37.1-56.2)$ \\
\hline & OCD & $10.7 \pm 6.2(1-22) \dagger, \dagger$ & $4.2 \pm 2.3(0-7) \ddagger$ & $50 \pm 23(17-86) \ddagger$ & $3.1 \pm 2.2(0.8-7.1) \dagger,+$ & $37.4 \pm 12.5(16.5-53.4)$ \\
\hline
\end{tabular}

The amount of tissue is in percentage points of the region of interest. All data are displayed as mean \pm SD (range), unless otherwise specified. Native samples received the maximum amount of points for score A and B $(*)$. Native samples did not display any unfilled area $(\S)$. $\dagger P<0.05$ (difference in same group between 3 and 6 months). $\$ P<0.05$ (compared to native)

advisable. The results of the present study suggest that this might be by preventing subchondral bone plate collapse by lowering edge loading through smaller diameter grafts [11], coupled to enhancement of the subchondral situation [25] in the form of scaffolds [24] to assist early articular coverage and to prevent irregular subchondral bone plate and cavitary lesion formation.

There are some limitations to our study design. First, although the ovine model was chosen due to its similarities in size, structure and reparative potential when compared to the human knee [30], our results may not be entirely generalized to humans. Second, we simultaneously performed an OATS procedure in the same knee, which may have influenced OCD healing. In humans, however, following a knee OATS procedure, a similar situation exists. Finally, immediate postoperative movement and subsequent weightbearing might have negatively influenced OCD healing. However, tibiofemoral joint contact forces in the quadruple sheep [36] are considerably lower than in humans. Also, by leaving one hind limb intact, animals may to some extent unload their operated limb [18]. This may resemble the clinical situation more closely, as in humans, postoperative non-weightbearing rehabilitation may be as short as 4 weeks $[29,37]$ and by 4 weeks, full recovery of the donor sites in humans has probably not taken place $[14,37]$.

This study confirms the complex and long healing process of large OCDs [14, 20, 25, 37]. Moreover, we found similar deleterious changes as in harvest sites of patients with concurrent donor site morbidity [37]. Therefore, in our opinion, orthopaedic surgeons performing OATS must critically consider the potential for degenerative changes following graft harvest. In particular, as pain amelioration is one of the main goals of the OATS procedure, caution may be warranted when selecting patients with known risk factors for donor site morbidity following OATS, such as higher body mass index [26]. 


\section{Conclusion}

Healing of the OCD donor site starts with restoration of the subchondral bone plate. However, at 6 months, subchondral bone plate restoration does not always result in closure of an artificially created OCD in the weightbearing part of an ovine femoral condyle. Moreover, subchondral cavitary lesion formation, subchondral bone and cartilage collapse, osseous erosion and degenerative changes in the reparative fibrocartilage and adjacent native cartilage were common findings. Osteochondral grafting in the OATS procedure must therefore be viewed critically.

Acknowledgments This study was supported by a grant of the German Research Foundation (DU 298/8-3). The authors would like to thank Mrs. G. Hardung, Mrs. M. Princ, Mrs. C. Bergmann and Mr. M. Wolny for the technical support.

Open Access This article is distributed under the terms of the Creative Commons Attribution Noncommercial License which permits any noncommercial use, distribution, and reproduction in any medium, provided the original author(s) and source are credited.

\section{References}

1. Ahmad CS, Cohen ZA, Levine WN et al (2001) Biomechanical and topographic considerations for autologous osteochondral grafting in the knee. Am J Sports Med 29:201-206

2. Allen MJ, Houlton JE, Adams SB, Rushton N (1998) The surgical anatomy of the stifle joint in sheep. Vet Surg 27:596-605

3. Braman JP, Bruckner JD, Clark JM et al (2005) Articular cartilage adjacent to experimental defects is subject to atypical strains. Clin Orthop Relat Res 430:202-207

4. Chen JH, Liu C, You L, Simmons CA (2010) Boning up on Wolff's law: mechanical regulation of the cells that make and maintain bone. J Biomech 43:108-118

5. Cox LGE, Lagemaat MW, van Donkelaar CC et al (2011) The role of pressurized fluid in subchondral bone cyst growth. Bone 49:762-768

6. Duda GN, Maldonado ZM, Klein P et al (2005) On the influence of mechanical conditions in osteochondral defect healing. J Biomech 38:843-851

7. Frenkel SR, Bradica G, Brekke JH et al (2005) Regeneration of articular cartilage - evaluation of osteochondral defect repair in the rabbit using multiphasic implants. Osteoarthr Cartil 13:798-807

8. Garretson RB III, Katolik LI, Verma N et al (2004) Contact pressure at osteochondral donor sites in the patellofemoral joint. Am J Sports Med 32:967-974

9. Gotterbarm T, Breusch SJ, Schneider U, Jung M (2008) The minipig model for experimental chondral and osteochondral defect repair in tissue engineering: retrospective analysis of 180 defects. Lab Anim 42:71-82

10. Guettler JH, Demetropoulos CK, Yang KH, Jurist KA (2005) Dynamic evaluation of contact pressure and the effects of graft harvest with subsequent lateral release at osteochondral donor sites in the knee. Arthroscopy 21:715-720

11. Guettler JH, Demetropoulos CK, Yang KH, Jurist KA (2004) Osteochondral defects in the human knee: influence of defect size on cartilage rim stress and load redistribution to surrounding cartilage. Am J Sports Med 32:1451-1458
12. Hangody L, Fules P (2003) Autologous osteochondral mosaicplasty for the treatment of full-thickness defects of weightbearing joints: ten years of experimental and clinical experience. J Bone Joint Surg Am 85-A(Suppl 2):25-32

13. Imade S, Kumahashi N, Kuwata S, Iwasa J, Uchio Y (2011) Effectiveness and limitations of autologous osteochondral grafting for the treatment of articular cartilage defects in the knee. Knee Surg Sports Traumatol Arthrosc. doi:10.1007/s00167-0111611-0

14. Jackson DW, Lalor PA, Aberman HM, Simon TM (2001) Spontaneous repair of full-thickness defects of articular cartilage in a goat model. A preliminary study. J Bone Joint Surg Am 83-A:53-64

15. Jakob RP, Franz T, Gautier E, Mainil-Varlet P (2002) Autologous osteochondral grafting in the knee: indication, results, and reflections. Clin Orthop Relat Res 401:170-184

16. Karataglis D, Green MA, Learmonth DJ (2006) Autologous osteochondral transplantation for the treatment of chondral defects of the knee. Knee 13:32-35

17. Kim SJ, Shin SJ (2000) Loose bodies after arthroscopic osteochondral autograft in osteochondritis dissecans of the knee. Arthroscopy 16:E16

18. Kleemann RU, Schell H, Thompson M et al (2007) Mechanical behavior of articular cartilage after osteochondral autograft transfer in an ovine model. Am J Sports Med 35:555-563

19. Kock NB, Smolders JM, Van Susante JL et al (2008) A cadaveric analysis of contact stress restoration after osteochondral transplantation of a cylindrical cartilage defect. Knee Surg Sports Traumatol Arthrosc 16:461-468

20. Madry H, Van Dijk CN, Mueller-Gerbl M (2010) The basic science of the subchondral bone. Knee Surg Sports Traumatol Arthrosc 18:419-433

21. Mainil-Varlet P, Rieser F, Grogan S et al (2001) Articular cartilage repair using a tissue-engineered cartilage-like implant: an animal study. Osteoarthr Cartil 9(Suppl A):S6-S15

22. McGahan PJ, Pinney SJ (2010) Current concept review: osteochondral lesions of the talus. Foot Ankle Int 31:90-101

23. Nishitani K, Shirai T, Kobayashi M et al (2009) Positive effect of alendronate on subchondral bone healing and subsequent cartilage repair in a rabbit osteochondral defect model. Am J Sports Med 37(Suppl 1):139S-147S

24. Panseri S, Russo A, Cunha C et al (2011) Osteochondral tissue engineering approaches for articular cartilage and subchondral bone regeneration. Knee Surg Sports Traumatol Arthrosc. doi: 10.1007/s00167-011-1655-1

25. Pape D, Filardo G, Kon E, Van Dijk CN, Madry H (2010) Disease-specific clinical problems associated with the subchondral bone. Knee Surg Sports Traumatol Arthrosc 18:448-462

26. Paul J, Sagstetter A, Kriner M et al (2009) Donor-site morbidity after osteochondral autologous transplantation for lesions of the talus. J Bone Joint Surg Am 91:1683-1688

27. Qiu YS, Shahgaldi BF, Revell WJ, Heatley FW (2003) Observations of subchondral plate advancement during osteochondral repair: a histomorphometric and mechanical study in the rabbit femoral condyle. Osteoarthr Cartil 11:810-820

28. Radin EL, Rose RM (1986) Role of subchondral bone in the initiation and progression of cartilage damage. Clin Orthop Rel Res 213:34-40

29. Reddy S, Pedowitz DI, Parekh SG et al (2007) The morbidity associated with osteochondral harvest from asymptomatic knees for the treatment of osteochondral lesions of the talus. Am J Sports Med 35:80-85

30. Rudert M (2002) Histological evaluation of osteochondral defects: consideration of animal models with emphasis on the rabbit, experimental setup, follow-up and applied methods. Cells Tissues Organs 171:229-240 
31. Schell H, Lienau J, Epari DR et al (2006) Osteoclastic activity begins early and increases over the course of bone healing. Bone 38:547-554

32. Schlichting K, Schell H, Kleemann RU et al (2008) Influence of scaffold stiffness on subchondral bone and subsequent cartilage regeneration in an ovine model of osteochondral defect healing. Am J Sports Med 36:2379-2391

33. Shapiro F, Koide S, Glimcher MJ (1993) Cell origin and differentiation in the repair of full-thickness defects of articular cartilage. J Bone Joint Surg Am 75:532-553

34. Shelbourne KD, Jari S, Gray T (2003) Outcome of untreated traumatic articular cartilage defects of the knee: a natural history study. J Bone Joint Surg Am 85-A(Suppl 2):8-16

35. Simonian PT, Sussmann PS, Wickiewicz TL et al (1998) Contact pressures at osteochondral donor sites in the knee. Am J Sports Med 26:491-494
36. Taylor WR, Ehrig RM, Heller MO et al (2006) Tibio-femoral joint contact forces in sheep. J Biomech 39:791-798

37. Valderrabano V, Leumann A, Rasch H et al (2009) Knee-to-ankle mosaicplasty for the treatment of osteochondral lesions of the ankle joint. Am J Sports Med 37(Suppl 1):105S-111S

38. van Dijk CN, Reilingh ML, Zengerink M, van Bergen CJ (2010) Osteochondral defects in the ankle: why painful? Knee Surg Sports Traumatol Arthrosc 18:570-580

39. Zengerink M, Struijs PA, Tol JL, van Dijk CN (2010) Treatment of osteochondral lesions of the talus: a systematic review. Knee Surg Sports Traumatol Arthrosc 18:238-246 